\title{
Announcement of B. John Davies Prize for the best paper published in IJAMT in 2017
}

\author{
Anthony Doyle ${ }^{1} \cdot$ Andrew Y. C. Nee ${ }^{2}$ \\ Published online: 15 December 2018 \\ (C) Springer-Verlag London Ltd., part of Springer Nature 2018
}

The B. John Davies Prize for the best paper published in IJAMT 2017 has been awarded to Jiang-qin Ge, Shi-ming $\mathrm{Ji}$, and Da-peng Tan. The prize recognizes exceptional articles published in IJAMT, and awards authors for making an especially significant contribution. The award was named after the late B. John Davies of the University of Manchester Institute of Science and Technology (UMIST), the founding editor-inchief of IJAMT who led the journal from its launch in 1985 until 2013.

\section{Process}

From all the papers received and published in 2017, five highly rated papers with recommendations by two or more reviewers for the B. John Davies Prize were shortlisted. The shortlisted papers were carefully scrutinized by the regional editors. The following paper ${ }^{1}$ has received the highest vote and has been awarded the 2017 prize.

\section{Title of paper}

A gas-liquid-solid three-phase abrasive flow processing method based on bubble collapsing

(The International Journal of Advanced Manufacturing Technology, 2018, Volume 95, Number 1-4, Page 1069)

\section{Authors}

Jiang-qin Ge 1, Shi-ming Ji 1, Da-peng Tan 1, 2

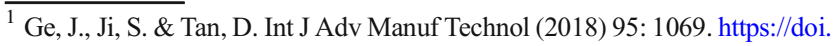
org/10.1007/s00170-017-1250-9

Anthony Doyle

anthony.doyle@springer.com

Andrew Y. C. Nee

mpeneeyc@nus.edu.sg

Springer, The Campus, 4 Crinan St, London N1 9XW, UK

2 National University of Singapore, Department of Mechanical Engineering, 9 Engineering Drive 1, Singapore 117576, Singapore

\section{Author affiliations}

1. Key Laboratory of E\&M, Ministry of Education \& Zhejiang Province, Zhejiang University of Technology, Hangzhou 310014, China; E-mail: tandapeng@ zjut.edu.cn; Tel/Fax: +86-571-88871052

2. State Key Laboratory of Digital Manufacturing Equipment \& Technology, Wuhan 430074, China

\section{Abstract}

Soft abrasive flow (SAF) processing presents advantages in avoiding surface damages and adapting complex workpiece shapes. However, the current SAF method exhibits low processing efficiency for hard-brittle materials. To solve this problem, a gas-liquid-solid three-phase abrasive flow processing method (GLSP) based on bubble collapsing is proposed. Through a surface constrained module, a multi-inlet constrained flow passage for silicon wafer processing is constructed, in which the bubbles are injected into the abrasive flow to strengthen the processing efficiency. On the basis of the Euler multi-phase model and population balance model (PBM), a GLSP fluid mechanic model is set up. Simulation results show that the bubble collapse region can be controlled by designing the flow passage structure, and that the near-wall particle turbulent motion can be strengthened by decreasing the fluid viscosity. The observation and processing experiments show that the most violent bubble collapsing occurs in the initial constrained surface region; Bubble collapsing can result in an average particle velocity increase from $12.90 \mathrm{~m} / \mathrm{s}$ to $15.97 \mathrm{~m} / \mathrm{s}$; the proposed GLSP method can increase the processing efficiency by $50 \%$ compared with the SAF method, and the average surface roughness can reach $2.84 \mathrm{~nm}$.

Anthony Doyle

Executive Editor, Springer

Andrew Y C Nee

Editor-in-Chief

Publisher's Note Springer Nature remains neutral with regard to jurisdictional claims in published maps and institutional affiliations. 